\title{
A New Method of Endoscopic Submucosal Dissection Using Submucosal Injection of Jelly
}

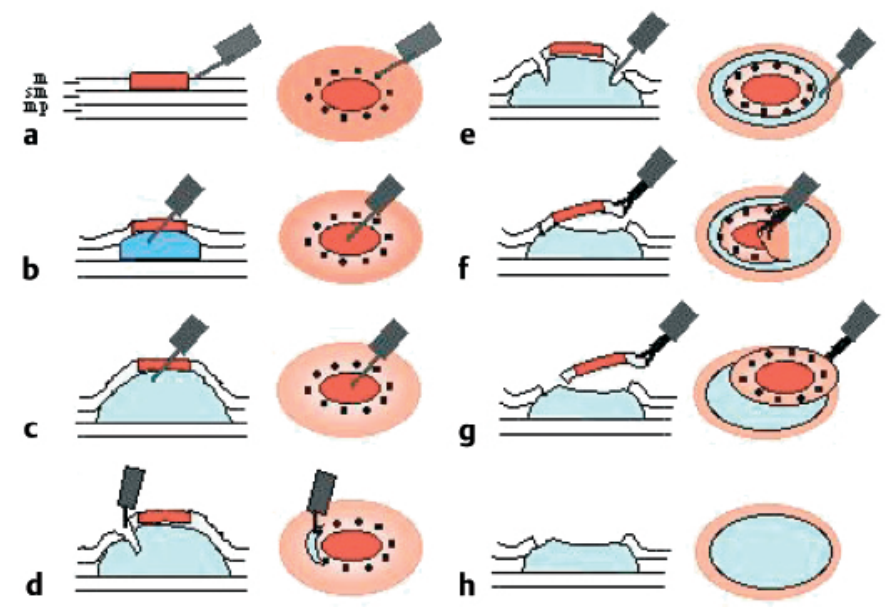

Figure 1 Endoscopic submucosal dissection with jelly injection (m, mucosa; sm, submucosa; $\mathrm{mp}$, muscularis propria). Dots are marked on the circumference of the lesion to outline the incision line (a). Physiological saline solution is first injected into the submucosa at the center of the lesion (b), and jelly is then injected into the submucosa at the center of the lesion (c). An initial mucosal incision is made using a needle-knife (d), and an incision is then made around the lesion using an insulated-tip knife (e). The lesion is held by grasping forceps (f) and pulled away from the muscular layer $(\mathbf{g})$. The lesion is resected completely, in one piece (h).

The technique of endoscopic submucosal dissection has been developed so that large lesions can be reliably resected en bloc. Several substances, including sodium hyaluronate, hydroxypropyl methylcellulose, and glycerol have been used to create a long-lasting submucosal fluid cushion [1 -4]. However, endoscopic submucosal dissection, and particularly the process of submucosal incision, is technically difficult and carries a high risk of perforation. We therefore report a new method of submucosal dissection by injecting jelly (Null Jelly; Teikoku Medix Co. Inc., Tokyo, Japan) into the submucosa. This method obviates the need for submucosal incision using a knife. The jelly, which is used as a sterilized medical lubricator in endoscopy, is thick and viscous. The elevation created by the jelly is significantly greater than that created by the other substances used for submucosal injection, and separates the submucosal layer from the muscular layer.
We performed endoscopic submucosal dissection with jelly injection in pigs as summarized in Figure 1. We marked dots on the circumference of the lesion to outline the margin. We first injected physiological saline solution at the center of the lesion to facilitate the injection of jelly into the submucosa, and then we injected the jelly at the center of the lesion using an 18-gauge needle and a $2.5-\mathrm{ml}$ syringe. We used an 18 -gauge needle and a $2.5-\mathrm{ml}$ syringe in order to minimize the resistance to injection because the jelly was very viscous. The total volume injected was determined by observing when mucosal elevation was sufficient to separate the submucosal layer from the muscular layer. After the jelly had been injected into the submucosa, we made an initial cut with a needle-knife and a mucosal incision around the lesion with an insulated-tip knife. Most of the submucosal lesion was dissected from the muscular layer by the injected jelly. We grasped

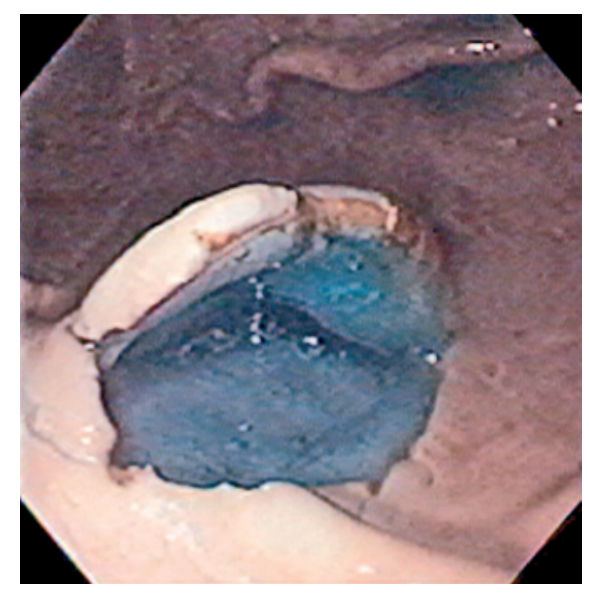

Figure 2 Endoscopic view of a pig's stomach, showing the incision around the lesion. Most of the submucosal lesion was dissected from the muscular layer by the injected jelly, which had been colored with Indigo carmine dye.

the lesion with a grasping forceps and pulled it away from the musucular layer (Figure 2). Submucosal incision with a knife was not required.

We resected five specimens in pigs' stomachs using this method. The average diameter of the specimens resected by submucosal injection of jelly was $40 \mathrm{~mm}$. There were no procedure-related complications. Endoscopic submucosal dissection with jelly injection appears to be an easy, safe, and technically efficient method for resecting gastric neoplasms.

\section{Yamasaki, K. Kume, K. Kanda, I. Yoshikawa, M. Otsuki}

Third Department of Internal Medicine, University of Occupational and Enviromental Health, School of Medicine, Kitakyusyu, Japan 
${ }^{1}$ Yamamoto $\mathrm{H}$, Yube $\mathrm{T}$, Isoda $\mathrm{N}$ et al. $\mathrm{A}$ novel method of endoscopic mucosal resection using sodium hyaluronate. Gastrointest Endosc 1999; 50: 251 - 256

2 Yamamoto H, Kawata H, Sunada K et al. Success rate of curative endoscopic mucosal resection with circumferential mucosal incision assisted by submucosal injection of sodium hyaluronate. Gastrointest Endosc 2002; 56: 507-512 ${ }^{3}$ Yamamoto H, Koiwai H, Yube T et al. A successful single-step endoscopic resection of a 40-millimeter flat-elevated tumor in the rectum: endoscopic mucosal resection using sodium hyaluronate. Gastrointest Endosc 1999; 50: 701 - 704
${ }^{4}$ Arnaldo B, Christopher J, Lawrence J et al. Hydroxypropyl methylcellulose: a better submucosal fluid cushion for endoscopic mucosal resection. Gastrointest Endosc 2003; 57: 41 - 47

Corresponding Author

\section{Otsuki, M. D.}

Third Department of Internal Medicine University of Occupational and Environmental Health
School of Medicine

1-1, Iseigaoka

Yahatanishi-ku

Kitakyusyu 807-8555

Japan

Fax: $\quad+81-93-692-0107$

E-mail: mac-otsk@med.uoeh-u.ac.jp 